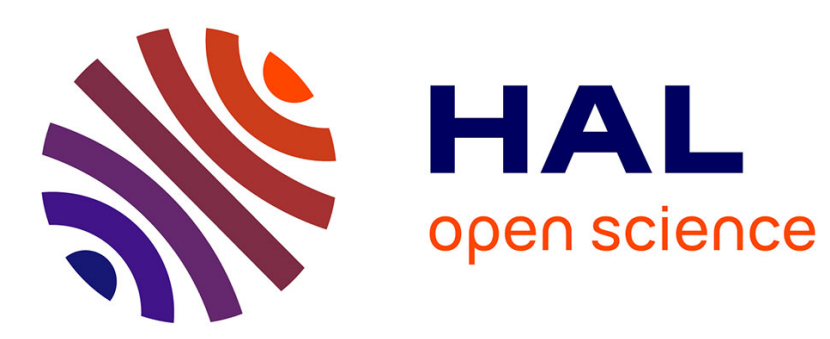

\title{
Antenne acoustique multi-éléments à focalisation. Modélisation du champ acoustique
}

\author{
J. Bresson, R. Barriol, J.P. Longuemard
}

\section{To cite this version:}

J. Bresson, R. Barriol, J.P. Longuemard. Antenne acoustique multi-éléments à focalisation. Modélisation du champ acoustique. Revue de Physique Appliquée, 1987, 22 (10), pp.1177-1184. 10.1051/rphysap:0198700220100117700 . jpa-00245667

\section{HAL Id: jpa-00245667 https://hal.science/jpa-00245667}

Submitted on 1 Jan 1987

HAL is a multi-disciplinary open access archive for the deposit and dissemination of scientific research documents, whether they are published or not. The documents may come from teaching and research institutions in France or abroad, or from public or private research centers.
L'archive ouverte pluridisciplinaire HAL, est destinée au dépôt et à la diffusion de documents scientifiques de niveau recherche, publiés ou non, émanant des établissements d'enseignement et de recherche français ou étrangers, des laboratoires publics ou privés. 
Classification

Physics Abstracts

$43.30-43.60-43.85-43.88$

\title{
Antenne acoustique multi-éléments à focalisation. Modélisation du champ acoustique
}

\author{
J. Bresson $\left({ }^{*}\right)$, R. Barriol $\left({ }^{*}\right)$ et J. P. Longuemard $\left({ }^{* *}\right)$ \\ (*) LMAI Université de Perpignan, avenue de Villeneuve, 66025 Perpignan, France \\ (**) LEPAP, Ecole Centrale des Arts et Manufactures, Grande Voie des Vignes, 92290 Châtenay-Malabry, \\ France
}

(Reçu le 24 décembre 1986, accepté le 18 juin 1987)

\begin{abstract}
Résumé. - La reconnaissance des couches superficielles du sédiment et éventuellement des objets enfouis, peut s'effectuer par moyens acoustiques. L'antenne de $0,5 \mathrm{~m}$ de rayon composée de 56 capteurs omnidirectionnels répartis sur 4 couronnes concentriques fonctionne en émission-réception à $46 \mathrm{kHz}$. Le traitement particulier en réception confère au système une bonne résolution angulaire et axiale avec des niveaux de lobes secondaires faibles. Ce résultat est obtenu par une focalisation électronique du faisceau acoustique procurant un contrôle aisé de la tache focale ainsi qu'une compensation de la défocalisation consécutive à la traversée d'un interface. Les auteurs présentent une modélisation du champ acoustique pour différentes configurations. Les résultats acquis en bassin confirment les possibilités prévues par le modèle.
\end{abstract}

\begin{abstract}
The recognition of superficial sédiment stratums or buried objects can be executed by acoustical methods. The half-meter radius tranducer array is made with 56 omnidirectionnal acoustic transducers. They are set on 4 concentric rings and they operate in emission reception at $46 \mathrm{kHz}$. A particular reception process gives axial and angular resolution to the system with low sidelobes levels. This result is obtained by an electronic focusing of the acoustic beam which gives an easy control of the focal spot and a compensation of the defocusing process related to the passage through an interface. The authors present a modelisation of acoustical field for various configurations. The acquisition of results in a water tank corroborates the possibilities provided by the model.
\end{abstract}

Différentes techniques permettent la reconnaissance de stratifications et d'objets enfouis dans les sédiments marins par la détection des ruptures d'impédances correspondantes. Les moyens mis en œuvre dépendent de la nature et de la dimension des milieux concernés $[1,2,5]$. Les auteurs présentent un procédé, compatible avec une épaisseur de sol sous-marin de l'ordre de quelques mètres, qui résulte d'un compromis entre la pénétration de l'onde et la définition recherchée.

Deux modes de fonctionnement sont envisagés : a) antenne au contact du fond, b) système fixé sur un vecteur navigant près du fond. Ces deux possibilités correspondent chacune à un modèle théorique particulier (avec ou sans interface entraînant l'existence d'un angle critique).

Le système proposé (Fig. 1) comporte un ensemble de transducteurs acoustiques omnidirectionnels répartis, dans un même plan, sur plusieurs couronnes concentriques [3, 9]. Par de judicieux déphasages introduits entre les signaux des couronnes, un front d'onde convergent est constitué, assurant ainsi une focalisation en un point de l'espace.

\section{Etude théorique.}

1.1 EMISSION ET FOCALISATION DANS LE MÊME MILIEU. - Ce cas correspond soit à une utilisation dans l'eau, ce qui est intéressant pour une eau fortement chargée (ou turbide), soit dans le sédiment. Le montage de 32 capteurs répartis sur 4 couronnes concentriques implique que le niveau acoustique en un point de l'espace résulte de la contribution de chacun des émetteurs ponctuels [4]. La focalisation électronique est obtenue en retardant électroniquement l'excitation des couronnes entre elles [7].

La pression de l'onde sphérique, issue d'un cap- 


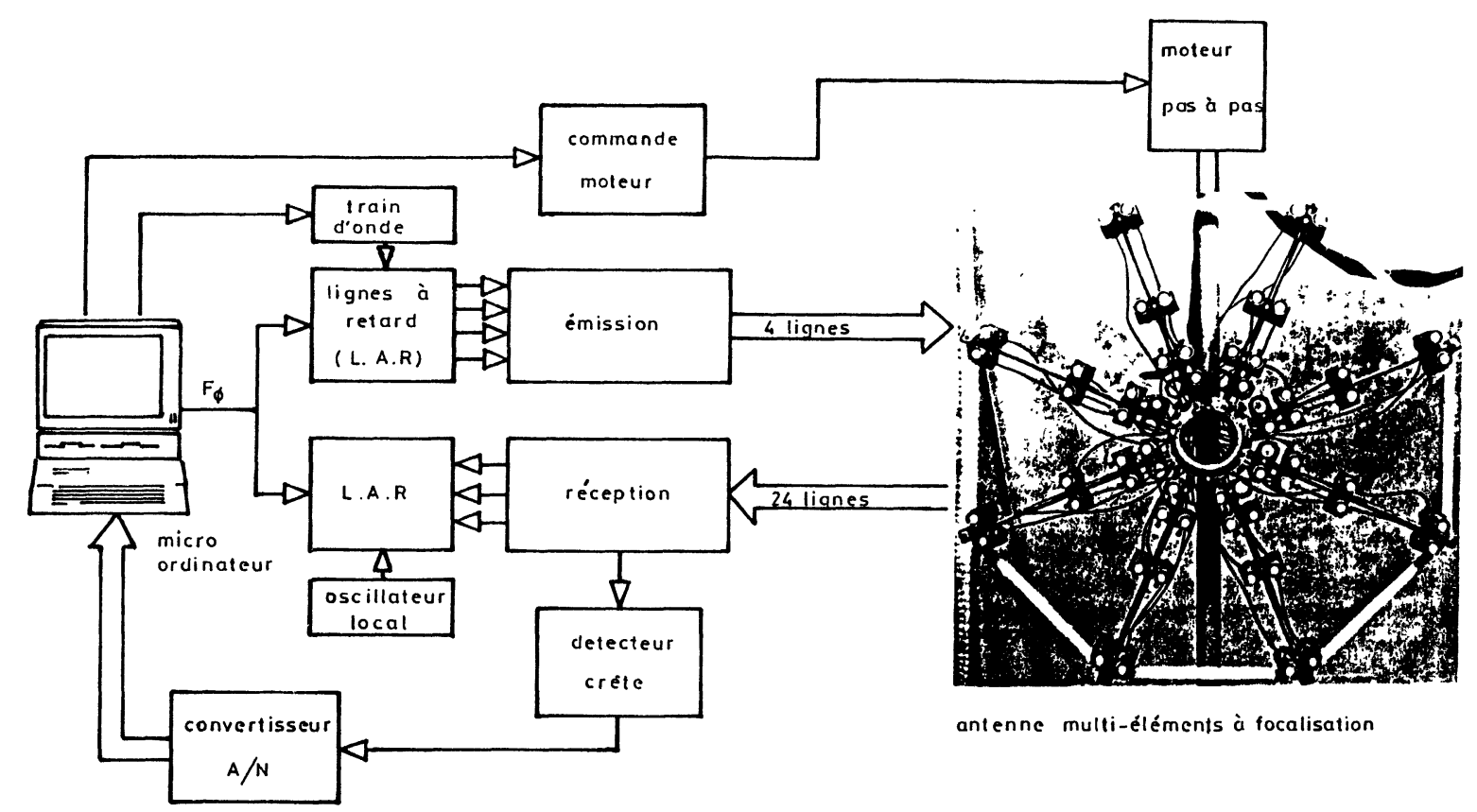

Fig. 1. - Schéma synoptique général de l'antenne multi-éléments à focalisation.

[Focusing transducers array block diagram.]

teur élémentaire $i$, reçue en un point $M$ de l'espace est de la forme :

$$
P_{i}=\frac{P_{0} r_{0}}{r_{i}} \cos \left(\omega t-k_{\mathrm{e}} r_{i}+\alpha_{\mathrm{ec}}\right)
$$

où $P_{0}$ est la pression créée par un capteur élémentaire à la distance $r_{0} ; r_{i}$ est la distance entre le capteur $i$ et le point $M, \alpha_{\mathrm{ec}}$ est le déphasage de la couronne à l'émission $\left(\alpha_{\mathrm{ec}}=\omega \tau_{\mathrm{ec}}\right)$ où $\tau_{\mathrm{ec}}$ est le retard introduit par la focalisation; $K_{\mathrm{e}}$ est le nombre d'onde dans l'eau égal à $k_{\mathrm{e}}=\frac{2 \pi}{\lambda}=\frac{\omega}{C_{\mathrm{e}}}$ ce qui se met aisément sous la forme d'une somme de sinus et cosinus

ou encore :

$$
P_{i}=A \cos \omega t+B \sin \omega t
$$

$$
P_{i}=X \cos (\omega t+\varphi)
$$

avec :

$$
\begin{aligned}
X & =\sqrt{A^{2}+B^{2}} \quad \varphi=\operatorname{arctg} \frac{B}{A} \\
A & =\frac{P_{0}}{r_{i}} \cos \left(k_{\mathrm{e}} r_{i}-\alpha_{\mathrm{ec}}\right) \\
B & =\frac{P_{0}}{r_{i}} \sin \left(k_{\mathrm{e}} r_{i}-\alpha_{\mathrm{ec}}\right) .
\end{aligned}
$$

Le modèle numérique mis au point permet de déterminer la pression provenant d'une couronne :

$$
P_{c}=\sum_{i=1}^{8} P_{i}
$$

avec le retard $\tau_{\mathrm{ec}}$ constant et par la suite, la détermination de la pression totale soit :

$$
P_{\Sigma}=\sum_{c=1}^{4} P_{c}
$$

l'on a introduit dans ce modèle divers retards entre les couronnes tels que la focalisation souhaitée soit obtenue.

Dans un premier temps, cette «loi de phases » a été déterminée en utilisant un schéma d'acoustique géométrique, qui visait à la génération d'une onde sphérique convergente à partir d'une antenne plane.

Ce modèle simple n'était pas tout à fait approprié car la position observée du maximum de la modulation de pression était plus proche de l'antenne que prévue par les calculs, et l'écart augmentait avec la distance de l'antenne.

Pour s'affranchir de cet effet imputable essentiellement à la divergence des ondes sphériques issues de chaque capteur, une autre méthode de calcul a été établie. Cette technique consiste, à partir des relations de l'acoustique physique précédentes, à rechercher par balayage numérique les valeurs des retards permettant l'obtention d'une réponse maximale au point de focalisation (Fig. 2).

Un problème similaire a été rencontré pour le fonctionnement en réception, et il a été de même nécessaire d'optimiser la « loi de phase » en fonction de la distance de focalisation.

L'exploitation de ce modèle avec des retards appropriés restitue le diagramme de rayonnement 


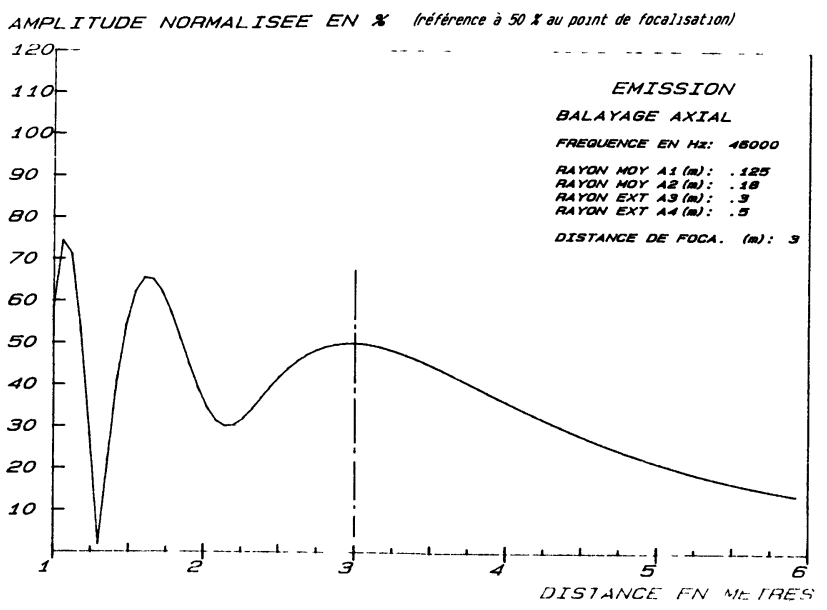

Fig. 2. - Tracé de la dorsale en émission pour la loi des retards calculés.

[Variation of axial pressure for emission with calculated delay times.]

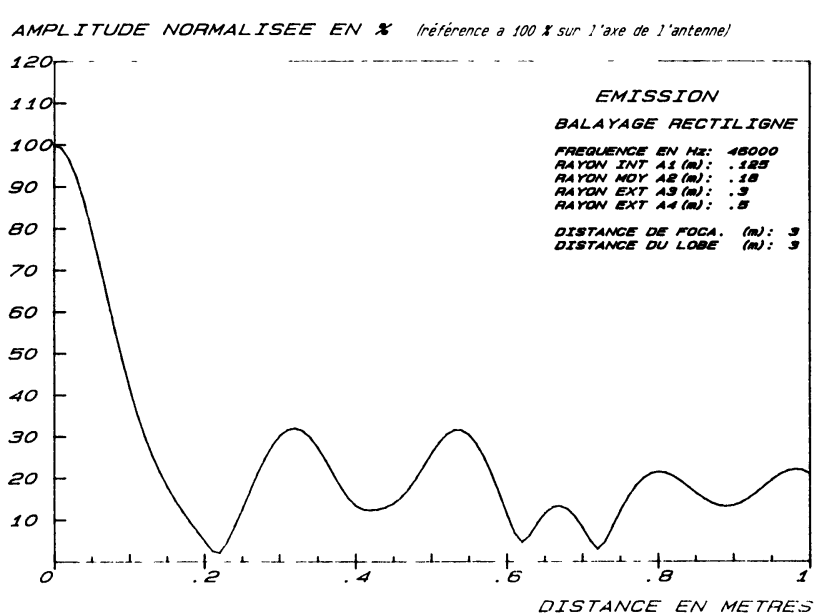

Fig. 3. - Diagramme de rayonnement en émission.

[Emission response pattern.]

en émission (Fig. 3) ; il est à remarquer que les lobes secondaires sont relativement importants.

1.2 RÉCEPTION ET FOCALISATION DANS LE MÊME MILIEU. - Il est classique de faire une opération de sommation des signaux issus des capteurs après avoir égalisé leurs phases. Cette opération s'identifie à une transformée de Fourrier discrète (TFD). Puis un traitement de pondération d'amplitude permet l'apodisation (suppression des lobes secondaires) de la réponse.

Pour cette étude, du fait de la symétrie axiale de l'antenne, nous avons préféré un traitement multiplicatif entre les signaux sommés puis égalisés issus des trois couronnes les plus externes [10].

L'apodisation s'effectue simplement en optimisant la position de la couronne médiane par rapport aux deux autres, et en considérant la complémentarité des diagrammes de rayonnement, qui en dehors du lobe principal ne présentent pas la même succession de maxima et de zéros. La multiplication des signaux permet alors de faire pratiquement disparaître les lobes secondaires.

Les résultats obtenus par ce traitement non linéaire, concernant le diagramme de rayonnement (niveau des lobes secondaires inférieur à $-20 \mathrm{~dB}$, Fig. 4), et la résolution axiale (Fig. 5), laissent espérer de bonnes caractéristiques de l'antenne en émission-réception.

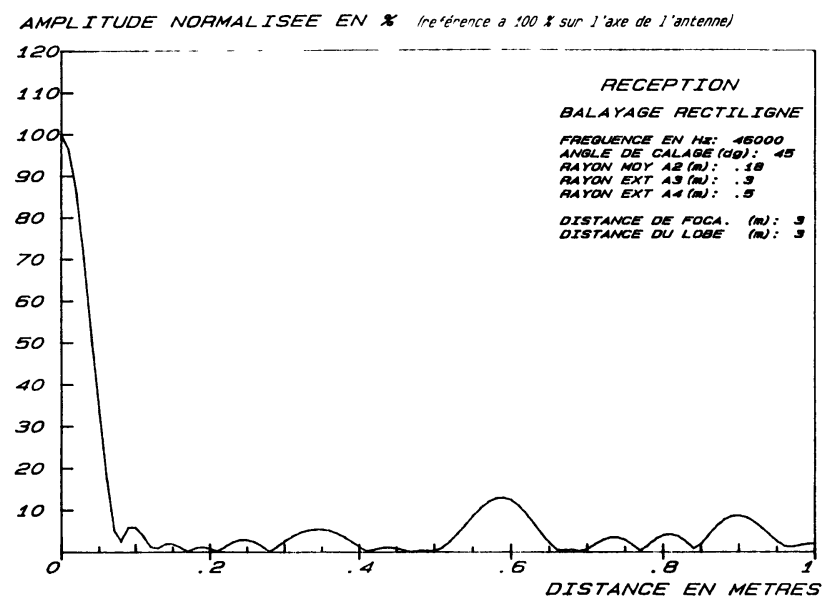

Fig. 4. - Diagramme de rayonnement en réception.

[Reception response pattern.]

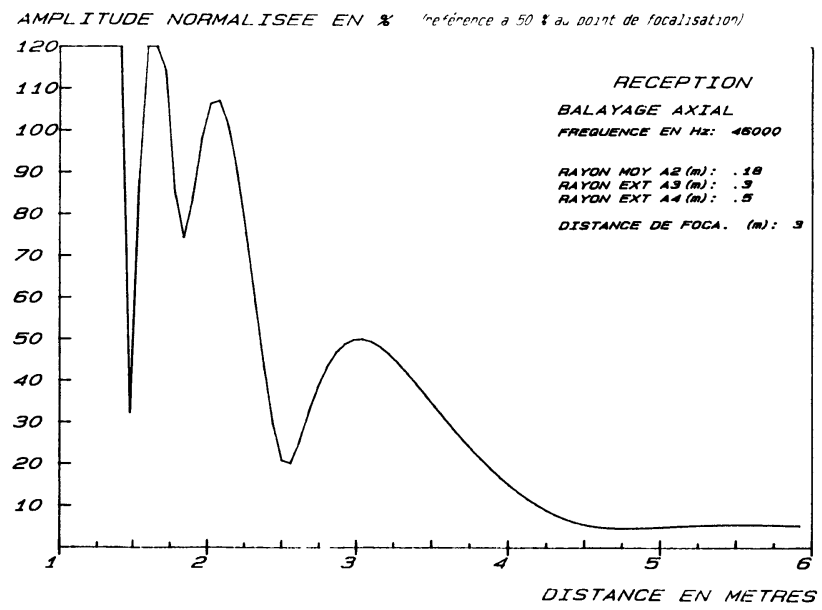

Fig. 5. - Tracé de la dorsale en réception.

[Variation of axial pressure for reception.]

La pression somme précédemment définie :

$$
P_{c}=\sum_{i=1}^{8} P_{i}=X_{c} \cos \left(\omega t+\beta_{c}\right)
$$


avec :

$$
\begin{aligned}
& X_{c}=\sqrt{A_{c}^{2}+B_{c}^{2}} \quad \beta_{c}=\gamma_{c}+\alpha_{\mathrm{rc}} \\
& \gamma_{c}=\operatorname{arctg} \frac{B_{c}}{A_{c}} \\
& A_{c}=\sum_{i=1}^{8} \frac{P_{0}}{r_{i}} \cos k_{\mathrm{e}} r_{i} \\
& B_{c}=\sum_{i=1}^{8} \frac{P_{0}}{r_{i}} \sin k_{\mathrm{e}} r_{i}
\end{aligned}
$$

où $\alpha_{\mathrm{rc}}$ est le déphasage de la couronne introduit par la focalisation à la réception, participe au résultat final de la façon suivante :

$$
\begin{aligned}
P_{\pi}=\prod_{c=1}^{3} P_{c}=\frac{1}{4}\left(\prod_{c=1}^{3} X_{c}\right) \times \\
\times \\
{\left[\cos \left(3 \omega t+\beta_{1}+\beta_{2}+\beta_{3}\right)\right.} \\
\left.+Y_{1} \cos \omega t-Y_{2} \sin \omega t\right]
\end{aligned}
$$

qui après développement donne :

$$
\begin{aligned}
P_{\pi}=\frac{1}{4}\left(\prod_{c=1}^{3} X_{c}\right) \cos \left(3 \omega t+\beta_{1}+\beta_{2}+\beta_{3}\right)+ \\
+\frac{1}{4}\left(\prod_{c=1}^{3} X_{c}\right) Y \cos (\omega t+\theta) .
\end{aligned}
$$

Le filtrage spatial centré sur le fondamental permet d'éliminer l'harmonique 3 et il ne reste plus qu'une onde sinusoïdale modulée en amplitude par le terme :

$$
P_{\text {amplitude }}=\frac{1}{4} X_{1} X_{2} X_{3} Y
$$

avec :

$$
\begin{gathered}
Y=\sqrt{Y_{1}^{2}+Y_{2}^{2}} \\
\theta=\operatorname{arctg} \frac{Y_{2}}{Y_{1}} \\
Y_{1}=\cos \left(\beta_{1}+\beta_{2}-\beta_{3}\right)+\cos \left(\beta_{2}+\beta_{3}-\beta_{1}\right)+ \\
+\cos \left(\beta_{3}+\beta_{1}-\beta_{2}\right) \\
Y_{2}=\sin \left(\beta_{1}+\beta_{2}-\beta_{3}\right)+\sin \left(\beta_{2}+\beta_{3}-\beta_{1}\right)+ \\
+\sin \left(\beta_{3}+\beta_{1}-\beta_{2}\right) .
\end{gathered}
$$

\subsection{EMISSION-RÉCEPTION ET FOCALISATION DANS} LE MÊME MILIEU. - Le diagramme de rayonnement correspondant au produit des fonctions de directivités en émission et réception (Fig. 6) fait apparâtre un lobe principal de 1 degré d'angle d'ouverture à $-3 \mathrm{~dB}$ et des lobes secondaires dont le niveau est inférieur de $-30 \mathrm{~dB}$ et ce pour une antenne de rayon extérieur de $0,5 \mathrm{~m}$ et pour une fréquence d'utilisation de $46 \mathrm{kHz}$. Il en est de même pour la longueur de la tache focale qui diminue (Fig. 7).

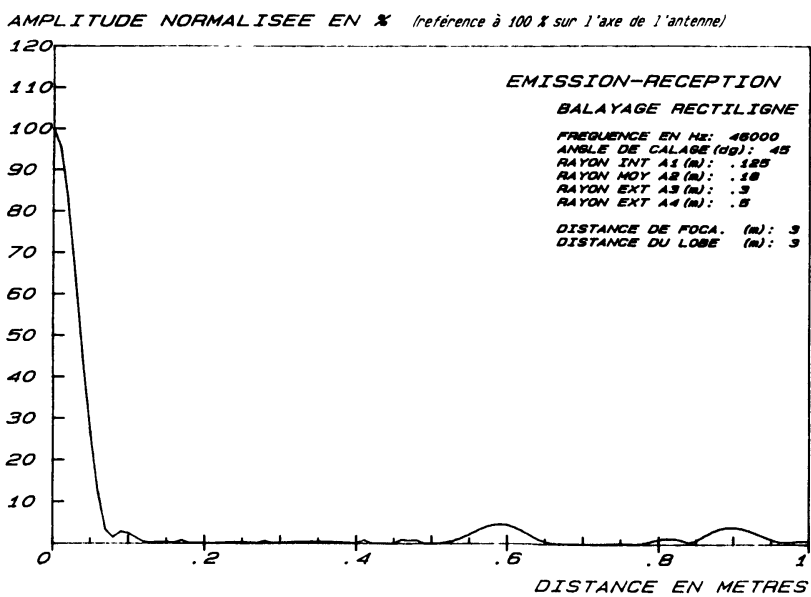

Fig. 6. - Diagramme de rayonnement en émission réception.

[Emission reception response pattern.]

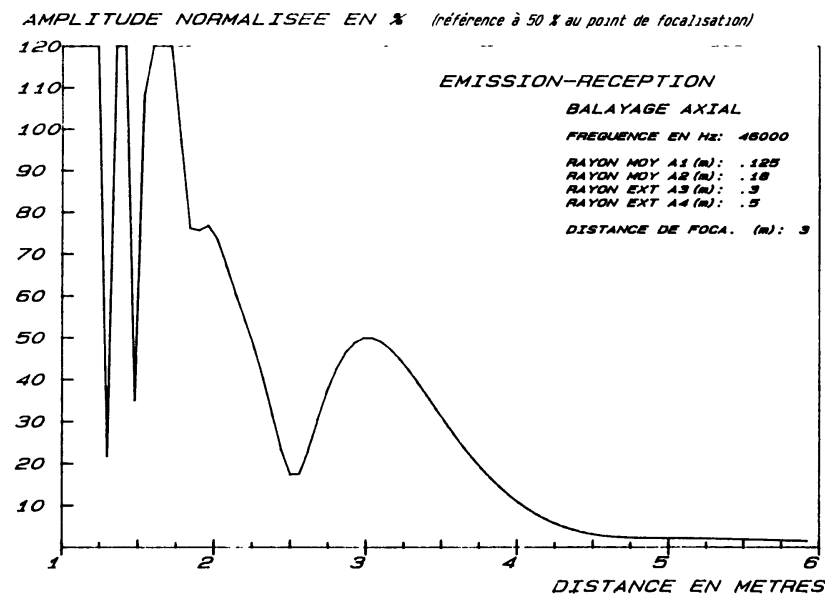

Fig. 7. - Tracé de la dorsale en émission réception.

[Variation of axial pressure for emission reception.]

1.4 FoCAlisation ET CHANGEMENT DE MILIEU. Divers phénomènes interviennent lors du passage d'une discontinuité acoustique : réflexion, réfraction, rétrodiffusion et diffusion. Dans cette étude, nous ne considèrerons que le comportement du faisceau acoustique réfracté ; ce dernier subit une modification liée à plusieurs paramètres (Fig. 8) :

- d'une part le faisceau subit une réfraction d'un angle $\alpha_{2}$ fonction des différents milieux ce qui entraîne un dépointage et une défocalisation de l'antenne,

- d'autre part la perte d'énergie de l'onde transmise apportée par la traversée de l'interface

$$
T=1-R=1-\left(\frac{Z_{\mathrm{s}} \cos \alpha_{1}-Z_{\mathrm{e}} \cos \alpha_{2}}{Z_{\mathrm{s}} \cos \alpha_{1}+Z_{\mathrm{e}} \cos \alpha_{2}}\right)^{2}
$$

et l'atténuation introduite par le caractère viscoélas- 


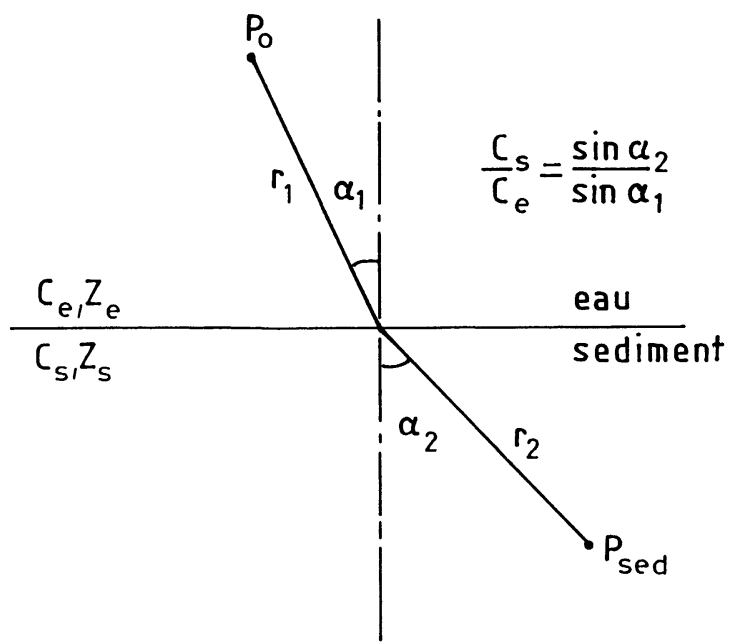

Fig. 8. - Réfraction du faisceau acoustique à la traversée d'un interface.

[Acoustical beam refraction to the passage through an interface.]

tique du sédiment limitent la pénétration du faisceau à quelques mètres. Les auteurs, dans leur majorité, proposent la loi d'atténuation en fonction de la fréquence de l'onde acoustique de la forme: $\alpha_{\mathrm{dB} \mathrm{m}^{-1}}=K F_{\mathrm{kHz}}^{n}$ où $n$ est voisin de 1 , et $K$ $\left(\mathrm{dB} \mathrm{m}^{-1} \mathrm{kHz}^{-1}\right)$ varie de 0,03 pour des sédiments vaseux à 1,68 pour des sables secs.

Cette atténuation dépend des caractéristiques sédimentologiques et géotechniques et semble augmenter avec la porosité pour les sables alors que le phénomène s'inverse pour les sédiments vaseux [6].

La modulation de pression provenant d'un transducteur élémentaire est maintenant donnée par l'expression :

$$
\begin{aligned}
P_{\text {séd. }}= & \frac{P_{0} r_{0}}{r_{1}+r_{2}}(1-R)^{1 / 2}+ \\
& \quad+\cos \left(\omega t-k_{\mathrm{e}} r_{1}-k_{\mathrm{s}} r_{2}+\alpha_{c}\right) \exp ^{-\delta_{\mathrm{s}} r_{2}}
\end{aligned}
$$

$r_{1}$ et $r_{2}$ sont les trajets acoustiques dans l'eau et dans le sédiment $\delta_{\mathrm{s}}=\alpha_{\mathrm{dB} \mathrm{m}^{-1} / 8,68}$ est l'atténuation $(\mathrm{Np} / \mathrm{m})$ apportée par le sédiment.

Pour le calcul de la divergence, du fait de la déformation des surfaces d'ondes (changement des courbures) lors du passage de l'interface, $r_{1}$ doit être pondéré par une fonction de l'incidence locale du faisceau à l'émission, il en sera de même de $r_{2}$ à la réception.

Une étude globale d'un modèle numérique faisant intervenir la totalité de ces paramètres est actuellement en cours, elle va permettre d'appréhender l'influence du second milieu sur les performances de l'antenne.

\section{Etude expérimentale.}

Les essais effectués dans une piscine de $6 \times 3 \times 2 \mathrm{~m}$ emplie d'eau confirment l'analyse théorique. Les données concernant le champ acoustique en émission (Fig. 9 et 10) pour différentes configurations (focalisation variable et point cible fixe, focalisation au point cible pour plusieurs distances) révèlent un niveau des lobes secondaires dans le meilleur des cas de $-10 \mathrm{~dB}$ avec un angle d'ouverture de 2,8 degrés à $-3 \mathrm{~dB}$.

Les mêmes mesures effectuées en réception dans les mêmes conditions, pour une distance de focalisation fixe (Fig. 11) donnent un angle d'ouverture de 1,6 degrés à $-3 \mathrm{~dB}$ et un niveau des lobes secondaires d'environ $-20 \mathrm{~dB}$.

La focalisation électronique consiste à introduire des retards entre les différentes couronnes d'une manière électronique.

Plusieurs procédés de lignes à retards ont été considérés, et nous avons retenu celui faisant appel à une technique hybride alliant souplesse d'utilisation, simplicité et faible coût [8].

Ce procédé fait appel à autant de portes analogiques (échantillonneurs analogiques) qu'il y a de signaux à retarder et à un seul registre à décalage numérique. Ce registre opère sur des trains de signaux carrés de fréquence $11,5 \mathrm{kHz}$; la fréquence de décalage $(250$ à $950 \mathrm{kHz})$ permet de contrôler la phase des signaux de sortie.

Selon le principe de l'hétérodynage, les signaux issus des couronnes $(46 \mathrm{kHz})$ sont mélangés avec les sorties du registre à décalage (qui ici font office d'oscillateurs locaux). Les phases des signaux résultant de ce battement dépendent ainsi de la fréquence de pilotage $f_{\varphi}$ du registre à décalage (et des numéros des sorties utilisées).

Les mesures effectuées selon ce procédé (Fig. 12) permettent de constater la qualité de l'apodisation : faible niveau des lobes secondaires, excepté à de très faibles distances de l'antenne (ce qui est inévitable mais sans inconvénient notoire).

On remarque aussi que la distance de focalisation varie linéairement avec la fréquence de pilotage du registre à décalage.

L'association des deux modes de fonctionnement (émission et réception) laisse espérer un accroissement des possibilités de détection de l'antenne.

\section{Conclusion.}

Les performances théoriques et réelles du système actuel, à savoir fréquence de fonctionnement $46 \mathrm{kHz}$ et rayon maximal $0,5 \mathrm{~m}$, permettent d'envisager une détection sur quelques mètres de sédiment et ceci avec une bonne résolution.

Le système de focalisation dynamique très souple, piloté par micro-ordinateur, pourra en fonction des conditions extérieures assurer automatiquement des 


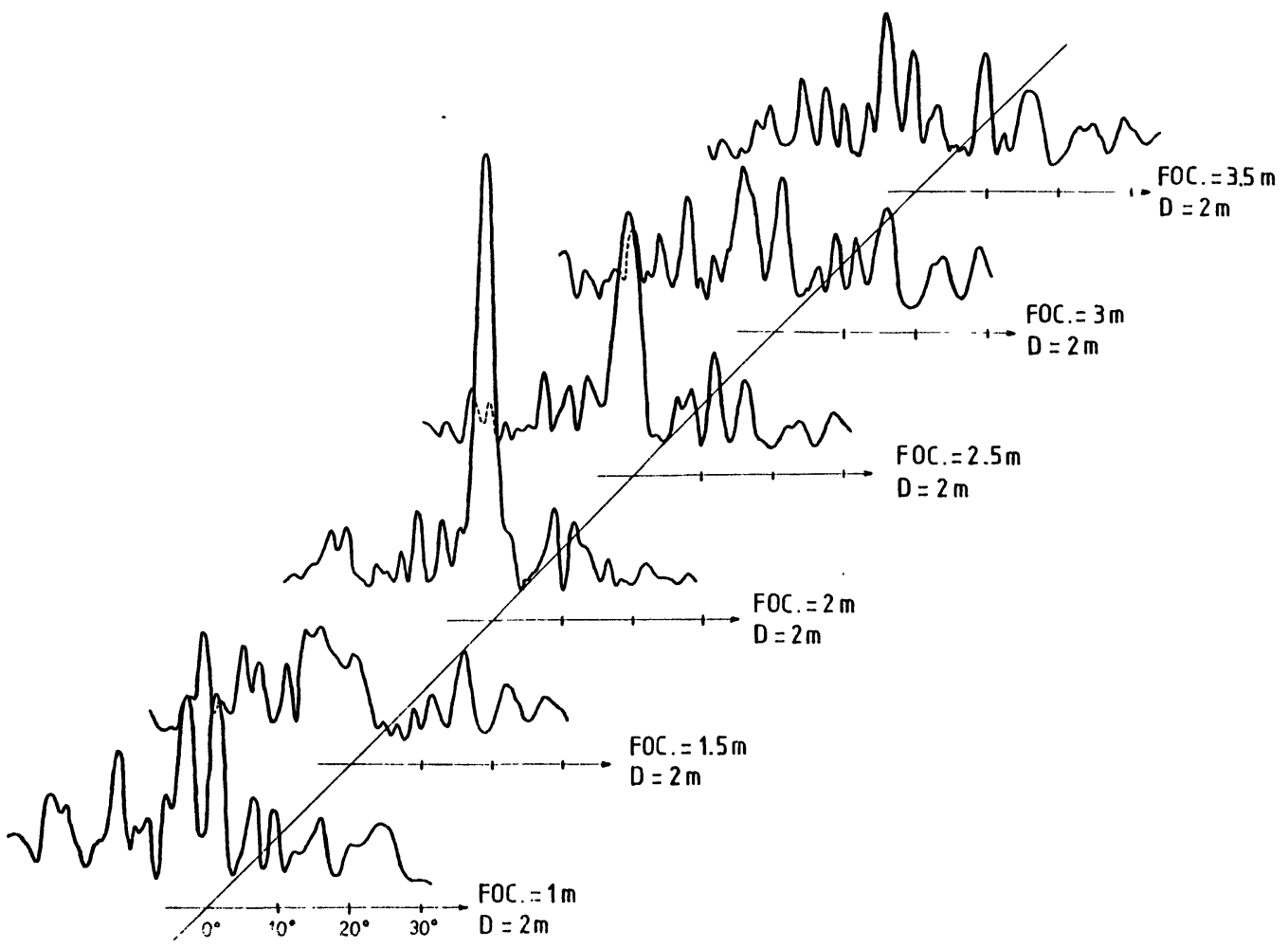

Fig. 9. - Relevé de champs acoustiques en émission de l'antenne à réseau (focalisation dynamique entre 1 et 3,5 m, point cible fixe à $2 \mathrm{~m}$ ).

[Acoustical fields experimentation of transducers array for emission (dynamical focusing from 1 to $3.5 \mathrm{~m}$ with a target at $2 \mathrm{~m})$.]

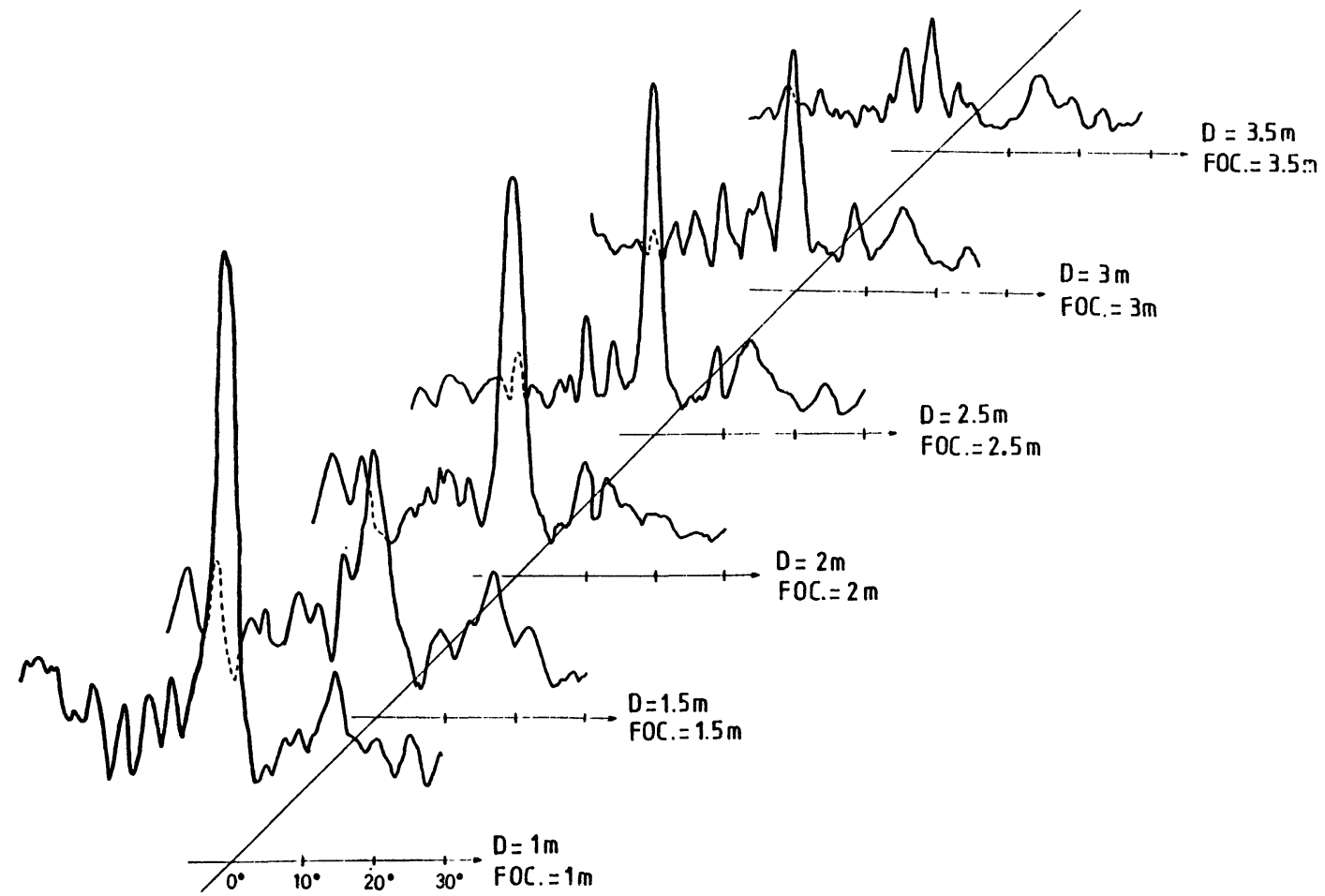

Fig. 10. - Relevé de champs acoustiques en émission de l'antenne à réseau pour différentes distances de focalisation. [Acoustical field measurements of the transducer array emission with various focal lengths.] 


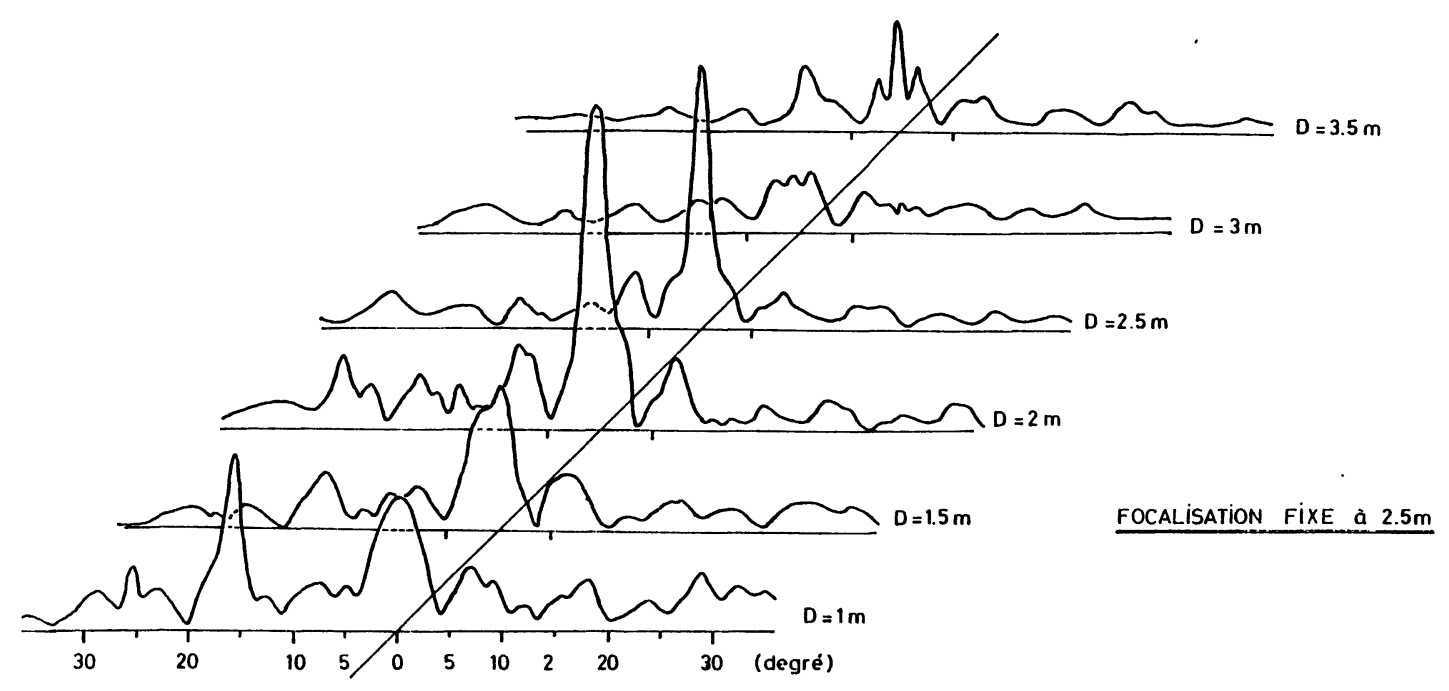

Fig. 11. - Relevé de diagrammes de rayonnement en réception entre 1 et $3,5 \mathrm{~m}$, pour une distance de focalisation de $2,5 \mathrm{~m}$.

[Response patterns for reception from 1 to $3.5 \mathrm{~m}$ with $2.5 \mathrm{~m}$ focusing length.]

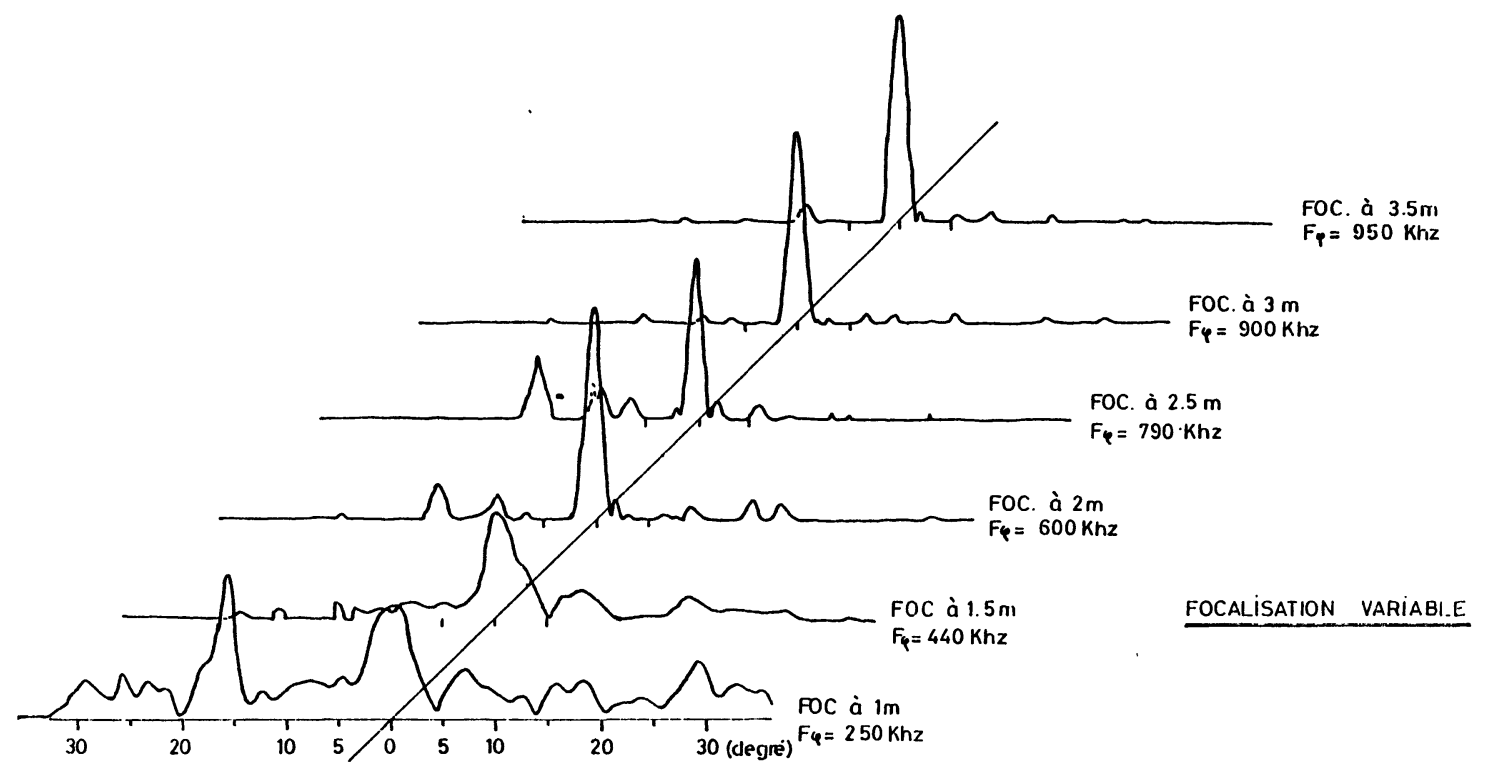

Fig. 12. - Diagrammes de rayonnement en réception pour différentes distances de focalisation.

[Response patterns for reception with various focusing lengths.]

corrections nécessaires afin d'optimiser la détection.

Les travaux actuels visent à la réalisation d'un dispositif d'imagerie acoustique pour une antenne supportée par un vecteur se déplaçant à proximité $\mathrm{du}$ fond à une vitesse de l'ordre de quelques mètres/seconde.

\section{Remerciements.}

Les auteurs remercient la DRET pour leur avoir permis par une aide financière d'étudier le principe ainsi que l'ANVAR qui leur a accordé une aide pour le développement. 


\section{Bibliographie}

[1] Alais, P., Imagerie et holographie ultrasonores. Revue Phys. Appl. 11 (5) (1976).

[2] Alais, P., Real time acoustical imaging with a $256 \times$ 256 matrix of electrostatic transducers. Acoust. Hologr. 5 (1974) 677-684.

[3] Bresson, J., Longuemard, J. P., Applications des caractéristiques géoacoustiques d'une argile artificielle à l'étude du champ acoustique d'une antenne multi-éléments. Revue Phys. Appl. 20 (1985).

[4] Brunell, C., Bridoux, E., Delannoy, B., NonGaillard, B., Rouvaen, J. M., TORguet, R., Effect of spatial sampling on an acoustical image reconstitution. J. Appl. Phys. 49 (1978).

[5] FInK, M., L'imagerie ultrasonore. Revue Phys. Appl. 18 (1983) 527-558.

[6] Hamilton, E. L., Compressional wave attenuation in marine sediments. Geophysics 37 (1972) 620646.
[7] Salvini, G., Calaora, A., Gazanhes, C., MorHANGE, J., Traitement d'antenne acoustique par une technique hybride. Huitième colloque sur le traitement du signal et ses applications (1981) 385-391.

[8] Salvini, G., Calaora, A., Gazanhes, C., Traitement d'antenne hybride. Neuvième colloque sur le traitement du signal et ses applications (1983) 811-815.

[9] Shibata, S., Koda, T., Matsumoto, S., Yamaga, J., Coaxial circular spherical array for ultrasonic imaging. J. Acoust. Soc. Amer. 62 (1977).

[10] Shibata, S., Koda, T., Yamaga, J., C-mode ultrasonic imaging by and electronically scanned coaxial circular spherical receiving array. Ultrasonic (1978) 65-68. 